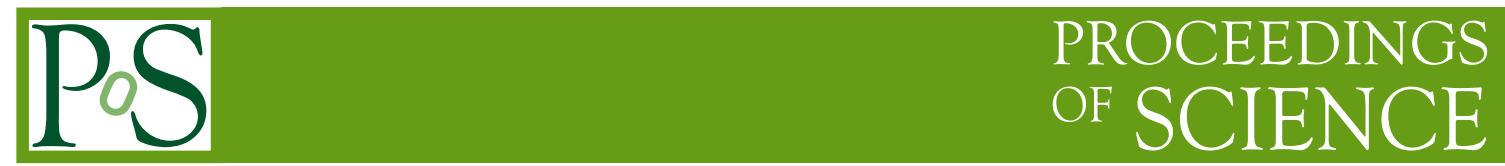

\title{
Inflation Physics from the CMB and LSS
}

\author{
Leonardo Senatore* \\ Stanford University \\ E-mail: senatoreastanford.edu
}

Inflation is the leading candidate for the earliest epoch of our universe. It consists of an early phase of de sitter like expansion that flattened the universe and produced the primordial fluctuations. How in detail did inflation happen? What is the Physics that governed that epoch? and, on a different topic, can we learn something on how inflation started? I will review our current knowledge of inflation and how we try to attack these questions through several observables in the CMB and large scale structures.

Frontiers of Fundamental Physics 14 - FFP14,

15-18 July 2014

Aix Marseille University (AMU) Saint-Charles Campus, Marseille

${ }^{*}$ Speaker. 